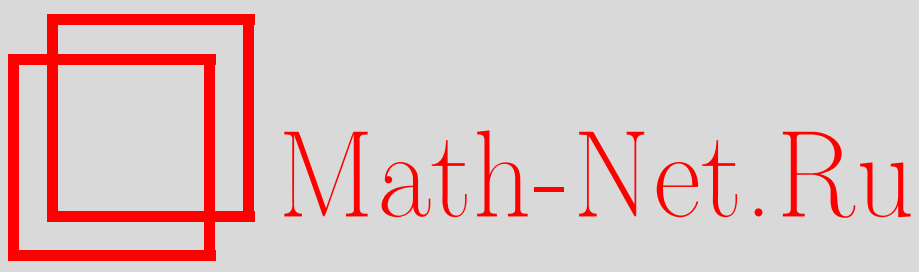

E. S. Kornev, The bundle of paracomplex structures, Sibirsk. Mat. Zh., 2020, Volume 61, Number 4, 867-879

DOI: https://doi.org/10.33048/smzh.2020.61.410

Use of the all-Russian mathematical portal Math-Net.Ru implies that you have read and agreed to these terms of use http://www . mathnet.ru/eng/agreement

Download details:

IP: 54.157 .27 .8

April 26, 2023, 14:18:05

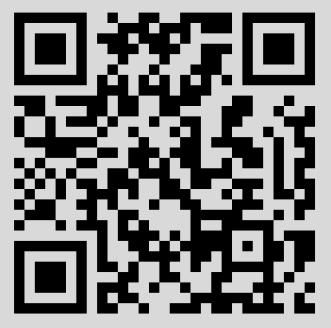


Сибирский математический журнал Июль-август, 2020. Том 61, № 4

УДК 514.763

\title{
РАССЛОЕНИЕ ПАРАКОМПЛЕКСНЫХ СТРУКТУР
}

\section{Е. С. Корнев}

\begin{abstract}
Аннотация. Рассматривается расслоение паракомплексных структур и связанные с ним проблемы существования на многообразии паракомплексной структуры. Получены явные описания расслоения паракомплексных структур для сфер размерности 2,4 и 6 . Доказано существование на шестимерной сфере неинтегрируемой почти паракомплексной структуры.
\end{abstract}

DOI $10.33048 /$ smzh.2020.61.410

Ключевые слова: паракомплексная структура, пара-кэлерова структура, расслоение паракомплексных структур, почти произведение.

\section{$\S 1$. Введение}

Почти паракомплексной структурой на многообразии $M$ размерности $2 n$ называют непрерывное поле автоморфизмов касательных пространств, квадрат которого представляет собой тождественный оператор, а собственные подпространства имеют размерность $n$. Понятие почти паракомплексной структуры является частным случаем понятия структуры почти произведения и является антиподом понятия почти комплексной структуры на многообразии. Для паракомплексных и пара-кэлеровых структур существуют результаты и объекты, аналогичные таким понятиям для почти комплексных структур, как интегрируемость, фундаментальная 2-форма, ассоциированная метрика и т. д. Наиболее полная информация об этом представлена в $[1,2]$. Для расслоения комплексных структур существует много известных результатов и описаний. Но для расслоения паракомплексных структур существует очень мало информации. В данной работе подробно определяется и описывается расслоение паракомплексных структур. В частности, показана связь расслоения ортогональных паракомплексных структур с расслоением грассманианов, а также описана структура расслоения ортогональных паракомплексных структур для четномерных сфер размерности 2,4 и 6 . Поскольку почти паракомплексную структуру на многообразии $M$ можно рассматривать как глобальное сечение расслоения паракомплексных структур над $M$, вопрос существования на многообразии почти паракомплексной структуры часто сводится к вопросу существования глобального сечения расслоения паракомплексных структур. Используя этот подход, мы покажем, что на четырехмерной сфере не существует даже неинтегрируемых почти паракомплексных структур, а на шестимерной сфере существует неинтегрируемая почти паракомплексная структура. Заметим, что в [3] получены почти паракомплексные структуры для шестимерных псевдосфер в псевдоевклидовом пространстве. В данной работе докажем существование неинтегрируемой почти паракомплексной структуры на стандартной шестимерной сфере в евклидовом пространстве.

(c) 2020 Корнев Е. C. 
В $\S 2$ дано определение почти паракомплексной структуры, а также приведены необходимые сведения для почти паракомплексных структур. В $§ 3$ дано определение расслоения ортогональных паракомплексных структур и показана связь этого расслоения с расслоением грассманианов. В $\S 4$ описано расслоение ортогональных паракомплексных структур над четырехмерной сферой и доказано, что это расслоение не допускает глобальных сечений. В $\S 5$ доказано существование на шестимерной сфере в евклидовом пространстве неинтегрируемой почти паракомплексной структуры с использованием 3-формы с нетривиальным радикалом по аналогии с тем, как это сделано в [4] для определения понятия субтвисторных и суб-кэлеровых структур с вырожденной фундаментальной 2-формой. Заметим, что если в [3] предъявлен явный вид почти паракомплексных структур для шестимерных псевдосфер, то в данной работе доказано только существование паракомплексной структуры на стандартной шестимерной сфере, но способов простого явного описания этой паракомплексной структуры нет.

\section{§ 2. Почти паракомплексные структуры}

Пусть $V$ - векторное пространство размерности $2 n$ над полем действительных чисел. Пусть $\Phi-$ невырожденный линейный оператор в $V$ такой, что $\Phi^{2}=\mathrm{id}$, где id - тождественный оператор. Из условия $\Phi^{2}=\mathrm{id}$ следует, что оператор $\Phi$ может иметь только два собственных значения: \pm 1 . Обозначим через $V_{+}$и $V_{-}$собственные подпространства, соответствующие собственным значениям 1 и -1 . В случае, когда $\operatorname{dim}\left(V_{+}\right)=\operatorname{dim}\left(v_{-}\right)=n$, оператор $\Phi$ называется паракомплексной структурой в векторном пространстве $V$. Простейшим примером паракомплексной структуры является линейный оператор в $V$, имеющий в фиксированном базисе матрицу

$$
\left(\begin{array}{cc}
\mathrm{id}_{n} & 0 \\
0 & -\mathrm{id}_{n}
\end{array}\right) .
$$

Если $M$ - гладкое многообразие размерности $2 n$, то для любой точки $x \in M$ в касательном пространстве $T_{x} M$ существует паракомплексная структура и $T_{x} M=V_{+}(x) \oplus V_{-}(x)$, где $V_{+}(x)$ и $V_{-}(x)$ - собственные подпространства паракомплексной структуры в касательном пространстве $T_{x} M$.

ОПРЕДЕЛЕНИЕ 2.1. Почти паракомплексной структурой на вещественном многообразии $M$ размерности $2 n$ называется непрерывное поле $\Phi$ автоморфизмов касательных пространств на $M$ такое, что $\Phi^{2}=\mathrm{id}$ и распределения собственных подпространств $V_{+}, V_{-}$суть непрерывные распределения ранга $n$ на $M$.

ЗАмЕчАниЕ 2.2. Почти паракомплексная структура на многообразии $M$ есть частный случай структуры почти произведения. Cmpуктурой почти произведения на $M$ называется непрерывное поле автоморфизмов касательных пространств на $M$, квадрат которого является полем тождественных операторов, а распределения собственных подпространств $V_{+}, V_{-}: T M=V_{+} \oplus V_{-}$имеют произвольный ранг.

Почти пара-эрмитовой структурой на многообразии $M$ размерности $2 n$ называется пара $(\Phi, h)$, где $h$ - псевдориманова метрика сигнатуры $(n, n)$ на $M, \Phi-$ почти паракомплексная структура на $M$ и $h \circ \Phi=-h$. Собственные подпространства $V_{+}(x)$ и $V_{-}(x)$ почти пара-эрмитовой структуры в любой точке $x \in M$ суть изотропные подпространства в касательном пространстве $T_{x} M$. 
ЗАмЕчАниЕ 2.3. Используя разбиение единицы, на любом паракомпактном многообразии размерности $2 n$ всегда можно построить псевдориманову метрику сигнатуры $(n, n)$. Если $q$ такая псевдориманова метрика, то любая почти паракомплексная структура $\Phi$ на $M$ вместе с псевдоримановой метрикой $h(X, Y)=q(X, Y)-q(\Phi X, \Phi Y), X, Y \in C^{1}(T M)$, образуют почти пара-эрмитову структуру на $M$.

Пусть $M$ - риманово многообразие четной размерности с римановой метрикой $g$. Почти паракомплексная структура $\Phi$ на $M$ называется ортогоналъной, если $g \circ \Phi=g$. Для ортогональной почти паракомплексной структуры распределения собственных подпространств $V_{+}$и $V_{-}$ортогональны относительно метрики $g$. Заметим, что если почти паракомплексная структура $\Phi$ не ортогональна относительно метрики $G$, то она ортогональна относительно метрики $h$ : $h(X, Y)=g(X, Y)+g(\Phi X, \Phi Y), X, Y \in C^{1}(T M)$. Таким образом, любая почти паракомплексная структура на римановом многообразии всегда ортогональна относительно некоторой римановой метрики.

Тензором кручения почти паракомплексной структуры $\Phi$ на многообразии $M$ называется тензорное поле $N$ типа $(2,1)$ такое, что для любых векторных полей $X, Y$ на $M$

$$
N(X, Y)=[\Phi X, \Phi Y]-\Phi[\Phi X, Y]-\Phi[X, \Phi Y]+[X, Y],
$$

где [.,.] обозначает скобку Ли векторных полей на $M$. Этот тензор является аналогом тензора Нейенхейса для почти паракомплексных структур.

На любом вещественном многообразии размерности $2 n$ в каждой координатной окрестности $U$ с локальными координатами $\left(x_{1}, \ldots, x_{n}, y_{1}, \ldots, y_{n}\right)$ существует локальная почти паракомплексная структура $\left.\Phi\right|_{U}$ :

$$
\Phi \frac{\partial}{\partial x_{k}}=\frac{\partial}{\partial x_{k}}, \quad \Phi \frac{\partial}{\partial y_{l}}=-\frac{\partial}{\partial y_{l}}
$$

где $\frac{\partial}{\partial x_{k}}, \frac{\partial}{\partial y_{l}}-$ локальные векторные поля, действующие на каждую гладкую функцию $f$ как частные производные функции $f$ по координатам $x_{k}, y_{l}$. Однако, обратно, не всегда для почти паракомплексной структуры $\Phi$ на многообразии $M$ существуют локальные координаты $\left(x_{1}, \ldots, x_{n}, y_{1}, \ldots, y_{n}\right)$, для которых выполнено условие (2). Почти паракомплексная структура на многообразии $M$ называется интегрируемой, или паракомплексной, если для каждой точки $x \in M$ существует координатная окрестность, в которой выполнено условие (2). Для почти паракомплексных структур получены следующие критерии интегрируемости (см. [1,2]).

Теорема 2.4. Пусть $\Phi-$ почти паракомплексная структура на гладком вещественном многообразии $M$ размерности $2 n$ и $N-$ ее тензор кручения. Тогда следующие условия эквивалентны:

(1) $\Phi-$ интегрируемая почти паракомплексная структура на $M$;

(2) распределения собственных подпространств $V_{+}, V_{-}$инволютивны;

(3) $N=0$ на $M$.

Пусть $M$ и $N$ - гладкие вещественные многообразия одинаковой размерности. На $M \times N$ всегда существует почти паракомплексная структура $\Phi$ : $\Phi x=\left.X\right|_{X \in C^{1}(T M)}, \Phi X=-\left.X\right|_{X \in C^{1}(T N)}$. Из теоремы 2.4 следует, что эта почти паракомплексная структура интегрируема. Таким образом получаем, что на прямом произведении гладких многообразий одинаковой размерности всегда 
существует интегрируемая почти паракомплексная структура. В частности, интегрируемая паракомплексная структура всегда существует на $S^{n} \times S^{n}, n \geq 1$, где $S^{n}-n$-мерная единичная сфера. Обратно, можно доказать следующий результат (см. [1,2]).

Теорема 2.5. Пусть $M$ - гладкое вещественное многообразие размерности 2n. Если на $M$ существует интегрируемая почти паракомплексная структура, то $M$ локально диффеоморфно прямому произведению двух $n$-мерных подмногообразий.

ЗАмЕЧАНИЕ 2.6. Часто почти паракомплексную структуру называют интегрируемой, если ее распределения собственных подпространств инволютивны. Из теоремы 2.4 следует, что это определение эквивалентно определению интегрируемости почти паракомплексной структуры, данному выше.

\section{§ 3. Расслоение паракомплексных структур}

В этом параграфе определено понятие расслоения паракомплексных структур над четномерным многообразием и показана его связь с расслоением грассманианов.

Пусть $M$ - гладкое вещественное многообразие размерности $2 n$ с римановой метрикой $g$. Для каждой точки $x \in M$ касательное пространство $T_{x} M$ есть векторное пространство размерности $2 n$ со скалярным произведением, индуцированным римановой метрикой $g$. Обозначим через $\mathscr{O}_{x}(M)$ множество всех ортогональных паракомплексных структур в $T_{x} M$. Для любой ортогональной паракомплексной структуры $\Phi_{x}$ в $T_{x} M$ определены собственные подпространства $V_{+}(x):\left.\Phi_{x}\right|_{V_{+}(x)}=\mathrm{id}$, и $V_{-}(x):\left.\Phi_{x}\right|_{V_{-}(x)}=-\mathrm{id}$. Поскольку подпространства $V_{+}(x)$ и $V_{-}(x)$ ортогональны и $T_{x} M=V_{+}(x) \oplus V_{-}(x)$, паракомплексная структура $\Phi_{x}$ полностью определяется выбором одного из этих подпространств. Для любого $n$-мерного подпространства $D(x) \subset T_{x} M$ существует единственное ортогональное дополнение $D^{\perp}(x)$. С подпространством $D(x)$ можно связать две паракомплексные структуры, а именно $\Phi_{x}:\left.\Phi_{x}\right|_{D(x)}=\mathrm{id},\left.\Phi_{x}\right|_{D^{\perp}(x)}=-\mathrm{id}$, и $-\Phi_{x}$ : $-\left.\Phi_{x}\right|_{D(x)}=-\mathrm{id},-\left.\Phi_{x}\right|_{D^{\perp}(x)}=\mathrm{id}$. Заметим, что при четном $n$ паракомплексная структура $\pm \Phi_{x}$ сохраняет ориентацию в $T_{x} M$, а при нечетном $n$ меняет ориентацию в $T_{x} M$. Таким образом, получаем

Предложение 3.1. Пусть $M$ - вещественное гладкое многообразие размерности $2 n$. Для любой точки $x \in M$ пространство $\mathscr{O}_{x}(M)$ есть тривиальное двулистное накрытие $n$-грассманиана в $\mathbb{R}^{2 n}$.

Обозначим через $\mathscr{O}(M)$ расслоение над гладким вещественным многообразием $M$ четной размерности, для которого существует гладкая проекция $\pi$ : $\mathscr{O}(M) \rightarrow M$ такая, что $\pi^{-1}(x)=\mathscr{O}_{x}(M)$ для любой точки $x \in M$. Будем называть это расслоение расслоением ортогональных паракомплексных струкmур. Поскольку на любом паракомпактном многообразии существует риманова метрика (см. [5,6]), расслоение ортогональных паракомплексных структур существует для любого паракомпактного многообразия. Таким образом, почти паракомплексную структуру на паракомпактном многообразии $M$ можно определить как глобальное сечение расслоения $\mathscr{O}(M)$. Здесь учтен полученный в $\S 2$ факт, что для любой почти паракомплексной структуры на римановом многообразии существует риманова метрика, относительно которой эта почти паракомплексная структура ортогональна. 
Обозначим через $\operatorname{gr}^{k} k$-грассманиан в $\mathbb{R}^{n}$, а через $\operatorname{gr}^{k}(M)$ - расслоение $k$ грассманианов над многообразием $M$. Из предложения 3.1 получаем

Следствие 3.2. Расслоение ортогональных паракомплексных структур над римановым многообразием $M$ размерности $2 n$ есть тривиальное двулистное накрытие расслоения $\operatorname{gr}^{n}(M)$.

Поскольку почти паракомплексная структура на паракомпактном многообразии $M$ является глобальным сечением расслоения $\mathscr{O}(M)$, из следствия 3.2 получаем критерий существования почти паракомплексной структуры.

Предложение 3.3. Паракомпактное многообразие $M$ размерности $2 n$ допускает почти паракомплексную структуру тогда и только тогда, когда на $M$ существует глобальное сечение расслоения $\mathrm{gr}^{n}(M)$.

Пусть $\mathrm{O}(k)$ - ортогональная группа евклидова пространства $\mathbb{R}^{k}$, а $\mathrm{U}(k)$ унитарная группа эрмитова пространства $\mathbb{C}^{k}$. Поскольку группа $\mathrm{O}(n)$ транзитивно действует на $k$-грассманиане в евклидовом пространстве $\mathbb{R}^{n}$, а подгруппа изотропии фиксированного $k$-мерного подпространства в $\mathbb{R}^{n}$ состоит из блочных матриц $\left(\begin{array}{cc}A & 0 \\ 0 & B\end{array}\right)$, где $A \in \mathrm{O}(k), B \in \mathrm{O}(n-k)$, получаем, что $\mathrm{gr}^{k} \cong \mathrm{O}(n) /(\mathrm{O}(k) \times \mathrm{O}(n-k))$ и $\operatorname{dim}\left(\mathrm{gr}^{k}\right)=k(n-k)$. Так как для риманова многообразия $M$ размерности $2 n$ слой расслоения $\mathscr{O}(M)$ есть двулистное накрытие $n$-грассманиана в пространстве $\mathbb{R}^{2 n}$ верно

Предложение 3.4. Пусть $\mathscr{O}(M)$ - расслоение ортогональных паракомплексных структур над римановым многообразием $M$ размерности $2 n$ с проекцией $\pi: \mathscr{O}(M) \rightarrow M$. Тогда $\pi^{-1}(x)$ для любой точки $x \in M$ является тривиальным двулистным накрытием грассманиана $\mathrm{gr}^{n} \cong \mathrm{O}(2 n) /(\mathrm{O}(n) \times \mathrm{O}(n))$ и $\operatorname{dim}\left(\pi^{-1}(x)\right)=n^{2}$.

Многообразие $M$ размерности $n$ называется параллелизуемым, если на $M$ существует глобальный $n$-репер. На параллелизуемом многообразии всегда существует риманова метрика, относительно которой глобальный $n$-репер на $M$ будет ортонормированным. Существование на параллелизуемом многообразии $M$ почти паракомплексной структуры равносильно тривиальности расслоения $\mathscr{O}(M)$.

Теорема 3.5. На вещественном параллелизуемом многообразии $M$ размерности $2 n$ существует почти паракомплексная структура тогда и только тогда, когда расслоение $\mathscr{O}(M)$ ортогональных паракомплексных структур над $M$ тривиально.

ДокАЗАтЕльство. Пусть на многообразии $M$ существует почти паракомплексная структура $\Phi$. Почти паракомплексная структура $\Phi$ определяет на $M$ пару распределений $n$-мерных касательных подпространств $D_{+}:\left.\Phi\right|_{D_{+}}=$ id, и $D_{-}:\left.\Phi\right|_{D_{-}}=-$id. Распределение $D_{+}$есть глобальное сечение расслоения $\operatorname{~gr}^{n}(M)$. Из предложения 3.4 следует, что для любой точки $x \in M n$ грассманиан в касательном пространстве $T_{x} M$ диффеоморфен $\mathrm{O}(2 n) /(\mathrm{O}(n) \times$ $\mathrm{O}(n))$. Будем считать, что $\mathbb{Z}_{2}= \pm 1$. Пусть $x \in M, A \in \mathrm{O}(2 n) /(\mathrm{O}(n) \times \mathrm{O}(n))$. Поскольку многообразие $M$ параллелизуемо, на $M$ существует глобальный $2 n$ репер $u$. В каждой точке $x \in M$ репер $u$ задает изоморфизм $\mathbb{R}^{2 n} \rightarrow T_{x} M$. Положим $A(v)=u \circ A \circ u^{-1}(v), v \in T_{x} M$. Получаем, что отображение $f:$ $f(x, A, 1)=A\left(D_{+}(x)\right), f(x, A,-1)=A\left(D_{-}(x)\right)$ есть изоморфизм расслоений $M \times \mathrm{O}(2 n) /(\mathrm{O}(n) \times \mathrm{O}(n)) \times \mathbb{Z}_{2}$ и $\mathscr{O}(M)$, т. е. расслоение $\mathscr{O}(M)$ тривиально. 
Поскольку любое тривиальное расслоение допускает стандартное глобальное сечение, если расслоение $\mathscr{O}(M)$ тривиально, из предложения 3.3 получаем, что на $M$ существует почти паракомплексная структура.

Пусть $M$ - паракомпактное многообразие размерности $n$ и $w_{k}(M)-k$-й класс Штифеля - Уитни многообразия $M$. Классы Штифеля - Уитни дают необходимое условие существования на многообразии глобального $k$-репера, $1 \leq$ $k \leq n$ (см. [7]).

Предложение 3.6. Пусть $M$ - паракомпактное многообразие размерности $n \geq 2$. Если на $M$ существует $k$ линейно независимых в каждой точке векторных полей, то $w_{n-i+1}(M)=0$ для всех $i: k \leq i \leq n+1$.

Рассмотрим двумерную единичную сферу $S^{2}$ в евклидовом пространстве $\mathbb{R}^{3}$. Глобальное сечение $s(x)$ расслоений 1-грассманианов на $S^{2}$ индуцирует векторное поле $V(x)$ на $S^{2}$, где $V(x)$ - единичный касательный вектор, порождающий касательную прямую $s(x)$ в точке $x$. Векторное поле $V(x)$ всюду отлично от 0 на $S^{2}$. Поскольку второй класс Штифеля - Уитни $w_{2}\left(S^{2}\right)$ порождает вторую группу когомологий $H^{2}\left(S^{2}, \mathbb{Z}_{2}\right) \cong \mathbb{Z}_{2}$, из предложений 3.3 и 3.6 получаем, что на $S^{2}$ не существует почти паракомплексной структуры.

Пусть $g_{0}$ - риманова метрика на $S^{2}$, индуцированная вложением $S^{2}$ в $\mathbb{R}^{3}$, и $P(2)$ - расслоение ортонормированных относительно метрики $g_{0}$ реперов на $S^{2}$. Пространство $P(2)$ состоит из упорядоченных пар $\left(e_{1}, e_{2}\right), e_{1}, e_{2} \in C^{1}\left(T\left(S^{2}\right)\right)$. Пусть $\pi_{1}$ - проекция $P(2) \rightarrow T\left(S^{2}\right): \pi_{1}\left(e_{1}, e_{2}\right)=e_{1}$, а $\pi_{2}-$ проекция касательного расслоения $T\left(S^{2}\right) \rightarrow S^{2}$. Для любой точки $x \in S^{2}$ и любого единичного касательного вектора $e_{1} \in T_{x}\left(S^{2}\right)$ в $T_{x}\left(S^{2}\right)$ существует только два единичных ортогональных $e_{1}$ вектора $\pm e_{2}$. Ортонормированный репер $\left(e_{1}, e_{2}\right)$ в точке $x$ определяет единственную ортогональную паракомплексную структуру $\Phi_{x}$ в $T_{x}\left(S^{2}\right)$ такую, что $\Phi_{x} e_{1}=e_{1}, \Phi_{x} e_{2}=-e_{2}$. Таким образом, пространство $P(2)$ вместе с проекцией $\pi_{1}$ является двулистным накрытием расслоения $\operatorname{gr}^{1}\left(S^{2}\right)$, а вместе с проекцией $\pi=\pi_{2} \circ \pi_{1}-$ расслоением над $S^{2}$. Тем самым имеет место

Теорема 3.7. Расслоение ортогональных паракомплексных структур над двумерной сферой $S^{2}$ изоморфно расслоению ортонормированных реперов над $S^{2}$ и не допускает глобального сечения.

ЗАмЕчАниЕ 3.8. Для двумерной сферы $S^{2}$ расслоение $\mathscr{O}\left(S^{2}\right)$ есть главное расслоение со структурной группой $\mathrm{O}(2)$. Это расслоение допускает редукцию к подрасслоению со структурной группой $\mathrm{SO}(2)$. Это подрасслоение изоморфно $T\left(S^{2}\right)$. Другими словами, касательное расслоение над $S^{2}$ является подрасслоением расслоения $\mathscr{O}\left(S^{2}\right)$.

\section{$\S$ 4. Расслоение паракомплексных структур над четырехмерной сферой}

Здесь описано расслоение ортогональных паракомплексных структур над четырехмерной сферой $S^{4}$. Известно, что на $S^{4}$ не существует почти комплексных структур (см. [4]), расслоение ортогональных комплексных структур, сохраняющих ориентацию (твисторное расслоение) на $S^{4}$, изоморфно комплексному проективному пространству $\mathbb{C} P^{3}$, а расслоение всех ортогональных комплексных структур над $S^{4}$ изоморфно $\mathbb{C} P^{3} \times \mathbb{Z}_{2}$ (см. [8]).

Рассмотрим четырехмерную единичную сферу $S^{4}$ в евклидовом пространстве $\mathbb{R}^{5}$ со стандартной евклидовой метрикой. Сферу $S^{4}$ можно отождествить 
с множеством пар $(q, a):|q|^{2}+|a|^{2}=1$, где $q$ - кватернион, $a$ - действительное число. Обозначим через $\mathbb{H}^{2}$ пространство упорядоченных пар кватернионов $\left(q_{1}, q_{2}\right)$. Считая, что мультипликативная группа ненулевых кватернионов $\mathbb{H}^{*}$ действует на $\mathbb{H}^{2}$ гомотетиями, получаем, что кватернионное проективное пространство $\mathbb{H} P^{1}$ диффеоморфно $\left(\mathbb{H}^{2} \backslash\{0\}\right) / \mathbb{H}^{*}$.

Зададим на $\mathbb{H}^{2} \backslash\{0\}$ функции

$$
f\left(q_{1}, q_{2}\right)=2 \frac{q_{1} \bar{q}_{2}}{\left|q_{1}\right|^{2}+\left|q_{2}\right|^{2}}, \quad h\left(q_{1}, q_{2}\right)=\frac{\left|q_{1}\right|^{2}-\left|q_{2}\right|^{2}}{\left|q_{1}\right|^{2}+\left|q_{2}\right|^{2}} .
$$

Поскольку $\left|f\left(q_{1}, q_{2}\right)\right|^{2}+\left|h\left(q_{1}, q_{2}\right)\right|^{2}=1$ для любой точки $\left(q_{1}, q_{2}\right) \in \mathbb{H}^{2} \backslash\{0\}$, отображение $\pi: \mathbb{H}^{2} \backslash\{0\} \rightarrow S^{4}, \pi\left(q_{1}, q_{2}\right)=\left(f\left(q_{1}, q_{2}\right), h\left(q_{1}, q_{2}\right)\right) \in \mathbb{R}^{5}$, есть накрывающее отображение $\mathbb{H}^{2} \backslash\{0\} \rightarrow S^{4}$. Так как отображение $\pi$ инвариантно относительно умножения на ненулевые кватернионы, оно является диффеоморфизмом $\mathbb{H} P^{1} \rightarrow S^{4}$.

Пусть $h_{0}$ - кватернионная метрика Фубини - Штуди на $\mathbb{H} P^{1}$ (см. [6]). ортогональной субкомплексной структурой в точке $x \in \mathbb{H} P^{1}$ называется пара $(D(x), J(x))$, где $D(x)$ - двумерное вещественное касательное подпространство в $T_{x}\left(\mathbb{H} P^{1}\right)$, а $J(x)$ - комплексная структура в векторном пространстве $D(x)$, ортогональная относительно метрики $h_{0}$. Более подробно о субкомплексных структурах см. в [4]. Поскольку в двумерном вещественном векторном пространстве всегда существуют только две ортогональные комплексные структуры (см. [5, гл. 9]), множество всех ортогональных субкомплексных структур в точке $x$ можно отождествить с $\mathrm{gr}^{2} \times \mathbb{Z}_{2}$, где $\mathrm{gr}^{2}-2$-грассманиан в $\mathbb{R}^{4}$. Применяя следствие 3.2 , получаем

Предложение 4.1. Расслоение $\mathscr{O}\left(S^{4}\right)$ ортогональных паракомплексных структур над четырехмерной сферой $S^{4}$ изоморфно расслоению $\operatorname{gr}^{2}\left(\mathbb{H} P^{1}\right) \times \mathbb{Z}_{2}$ ортогональных субкомплексных структур над $\mathbb{H} P^{1}$ со слоем $\mathrm{O}(4) /(\mathrm{O}(2) \times \mathrm{O}(2)) \times$ $\mathbb{Z}_{2}$.

Теперь докажем, что расслоение $\mathscr{O}\left(S^{4}\right)$ не допускает глобальных сечений.

Предложение 4.2. Расслоение ортогональных паракомплексных структур над четырехмерной сферой не допускает глобальных сечений.

ДоказАтЕльство. Предположим, что на $S^{4}$ существует почти паракомплексная структура $\Phi$. Поскольку на римановом многообразии любая почти паракомплексная структура ортогональна относительно некоторой римановой метрики (см. $\S 2)$, можно считать, что $\Phi$ - глобальное сечение расслоения $\mathscr{O}\left(S^{4}\right)$. Пусть $D_{+}$и $D_{-}-$распределения собственных подпространств для почти паракомплексной структуры $\Phi$. Имеем $\operatorname{rank}\left(D_{+}\right)=\operatorname{rank}\left(D_{-}\right)=2, T\left(S^{4}\right)=$ $D_{+} \oplus D_{-}$.

Пусть $p(E)$ - полный класс Понтрягина векторного расслоения $E$, а $p_{i}(E)-$ $i$-й класс Понтрягина векторного расслоения $E$. Поскольку $D_{+}$и $D_{-}-$векторные расслоения ранга 2, имеем $p_{1}\left(D_{+}\right)=p_{1}\left(D_{-}\right)=0$. Используя свойства характеристических классов для суммы Уитни векторных расслоений (см. [7]), получаем

$1+p_{1}\left(T\left(S^{4}\right)\right)=p\left(T\left(S^{4}\right)\right)=p\left(D_{+}\right) \smile p\left(D_{-}\right)=\left(1+p_{1}\left(D_{+}\right)\right) \smile\left(1+p_{1}\left(D_{-}\right)\right)=1$,

откуда $p_{1}\left(T\left(S^{4}\right)\right)=0$. С другой стороны, первый класс Понтрягина четырехмерной сферы $S^{4}$ порождает четвертую группу когомологий $H^{4}\left(S^{4}, \mathbb{Z}\right) \cong \mathbb{Z}$, а 
следовательно, не может быть нулевым. Это доказывает, что расслоение $\mathscr{O}\left(S^{4}\right)$ не допускает глобальных сечений.

ЗАмЕЧАНИЕ 4.3. Поскольку любое тривиальное расслоение допускает глобальное сечение, из предложения 4.2 следует, что расслоение $\mathscr{O}\left(S^{4}\right)$ нетривиально.

Из предложений 3.3 и 4.2 получаем

Следствие 4.4. На четырехмерной сфере не существует почти паракомплексных структур.

ЗАмЕЧАНИЕ 4.5. Поскольку вложение группы $\mathrm{O}(2) \times \mathrm{O}(2)$ в группу $\mathrm{O}(4)$ не является подгруппой в $\mathrm{SO}(4)$, не существует редукции расслоения $\mathscr{O}\left(S^{4}\right)$ к подрасслоению со слоем $\mathrm{SO}(4) /(\mathrm{O}(2) \times \mathrm{O}(2)) \times \mathbb{Z}_{2}$.

Вложение группы $\mathrm{SO}(2) \times \mathrm{SO}(2)$ в группу $\mathrm{SO}(4)$ есть подгруппа в $\mathrm{SO}(4)$. Отождествим евклидово пространство $\mathbb{R}^{4}$ с кватернионным пространством $\mathbb{H}$, а евклидову метрику в $\mathbb{R}^{4}-$ с метрикой $g_{0}$ в $\mathbb{H}$, где $g_{0}(x, y)=\operatorname{Re}(x \bar{y}), x, y \in \mathbb{H}$. Пусть $A\left(q_{1}, q_{2}\right)$ - линейное отображение $\mathbb{H} \rightarrow \mathbb{H}$ такое, что $A\left(q_{1}, q_{2}\right) x=q_{1} x \bar{q}_{2}$, $\left|q_{1}\right|=\left|q_{2}\right|=1$, для любого $x \in \mathbb{H}$. Легко проверить, что отображение $A\left(q_{1}, q_{2}\right)$ сохраняет метрику $g_{0}$, т. е. является ортогональным оператором в $\mathbb{H}$. Множество всех таких ортогональных операторов образует связную группу Ли относительно операции композиции, которая изоморфна группе $\mathrm{SO}(4)$. Заметим, что $A\left(-q_{1},-q_{2}\right)=A\left(q_{1}, q_{2}\right)$, откуда следует, что $\mathrm{SO}(4) \cong\left(S^{3} \times S^{3}\right) / \pm 1$. Будем считать, что группа $S^{1} \times S^{1}$ действует на $S^{3} \times S^{3}$ следующим образом:

$(a, b)\left(q_{1}, q_{2}\right)=\left(a q_{1}, b q_{2}\right),(a, b) \in \mathbb{C}^{2}, \quad|a|=|b|=1,\left(q_{1}, q_{2}\right) \in \mathbb{H}^{2}, \quad\left|q_{1}\right|=\left|q_{2}\right|=1$.

Так как $\mathrm{SO}(2) \cong S^{1}, S^{3} / S^{1} \cong \mathbb{C} P^{1}$, имеем

$$
\begin{aligned}
\mathrm{SO}(4) /(\mathrm{SO}(2) \times \mathrm{SO}(2)) & \cong\left(S^{3} \times S^{3}\right) /\left( \pm 1\left(S^{1} \times S^{1}\right)\right) \\
& \cong\left(S^{3} \times S^{3}\right) /\left(S^{1} \times S^{1}\right) \cong\left(S^{3} / S^{1}\right) \times\left(S^{3} / S^{1}\right) \cong \mathbb{C} P^{1} \times \mathbb{C} P^{1} .
\end{aligned}
$$

Теперь получаем

Предложение 4.6. Расслоение $\mathscr{O}\left(S^{4}\right)$ допускает редукцию к подрасслоению со слоем $\mathbb{C} P^{1} \times \mathbb{C} P^{1}$.

ЗАмечАниЕ 4.7. Поскольку $\mathbb{C} P^{1}$ диагонально вкладывается в $\mathbb{C} P^{1} \times \mathbb{C} P^{1}$, расслоение $\mathscr{O}\left(S^{4}\right)$ допускает редукцию к подрасслоению со слоем $\mathbb{C} P^{1}$. Однако это подрасслоение неизоморфно твисторному расслоению над $S^{4}$.

\section{$\S 5$. Почти паракомплексные структуры на шестимерной сфере}

Известно, что на шестимерной сфере существует неинтегрируемая почти комплексная структура (см. [5, гл. 9]). Здесь будет доказано, что на шестимерной сфере существует неинтегрируемая почти паракомплексная структура. В силу замечания 2.6 здесь будем называть почти паракомплексную структуру интегрируемой, если ее распределения собственных подпространств инволютивны.

Пусть $S^{6}$ - единичная шестимерная сфера в $\mathbb{R}^{7}$. Отождествим пространство $\mathbb{R}^{7}$ с алгеброй Клиффорда над $\mathbb{R}^{4}$. Такая алгебра Клиффорда состоит из октонионов $h=\sum_{k=1}^{7} a_{k} i_{k}$, где $a_{k}$ - действительное число, $i_{k}^{2}=-1, i_{k} i_{l}=-i_{l} i_{k}$, 
$\underline{i}_{1} i_{2}=i_{3}, i_{1} i_{4}=i_{5}, i_{2} i_{4}=i_{6}, i_{1} i_{6}=i_{7}$. Сопряжение октониона $h$ есть октонион $\bar{h}=-h$, а умножение октонионов - умножение в алгебре Клиффорда. Пусть $g_{0}$ - стандартная евклидова метрика в $\mathbb{R}^{7}$. Для любых октонионов $x, y$ имеем $g_{0}(x, y)=\operatorname{Re}(x \cdot \bar{y})$. Определим в $\mathbb{R}^{7} 3$-форму $\beta(x, y, z)=g_{0}(x \cdot \bar{y}, z)$. Обозначим через $G_{2}$ группу всех ортогональных линейных преобразований $\mathbb{R}^{7}$, сохраняющих 3 -форму $\beta$. Известно, что группа $G_{2}$ есть простая компактная группа Ли размерности 14. Имеем

$$
S^{6}=\left\{h \in \mathbb{R}^{7}: g_{0}(h, h)=1\right\} .
$$

Группа $G_{2}$ транзитивно действует на $S^{6}$, а подгруппа изотропии точки $i_{4}$ изоморфна $\mathrm{SU}(3)$ (см. $[5,6])$. Таким образом, сфера $S^{6}$ является однородным пространством $G_{2} / \mathrm{SU}(3)$.

Пусть $M=G / H$ - вещественное однородное риманово пространство размерности $2 n$, где $G$ - группа изометрий, транзитивно действующая на $M$, $H$ - подгруппа изотропии начальной точки $o \in M$. Почти паракомплексная структура $\Phi$ на $M$ называется $G$-инвариантной, если $\Phi_{x} \circ d g=d g \circ \Phi_{o}$, $x=g(o)$ для любого $g \in G$, где $d g$ - дифференциал отображения $g$. Любая $G$-инвариантная почти паракомплексная структура на $M$ однозначно определяется парой $G$-инвариантных распределений $n$-мерных касательных подпространств $D_{+}, D_{-}$на $M$, где $\left.\Phi\right|_{D_{+}}=\mathrm{id},\left.\Phi\right|_{D_{-}}=-\mathrm{id}$. Для ортогональной $G$-инвариантной почти паракомплексной структуры на $M$ достаточно задать только распределение $D_{+}$, так как распределение $D_{-}$всегда можно выбрать как ортогональное дополнение к $D_{+}$. Таким образом, задача получения на $M$ $G$-инвариантной ортогональной почти паракомплексной структуры сводится к получению на $M G$-инвариантного распределения $n$-мерных касательных подпространств.

Кососимметричная $p$-форма на однородном римановом пространстве $M=$ $G / H$ называется $G$-инвариантной, если $\Omega \circ d g=\Omega$ для любого $g \in G$. Любая $G$ левоинвариантная $H$-правоинвариантная $p$-форма на группе Ли $G$ индуцирует $G$-инвариантную $p$-форму на однородном пространстве $M$ (см. [6]).

ОПРЕДЕЛЕНИЕ 5.1. Радикалом р-формъ $\Omega$ на многообразии $M$ называется распределение касательных подпространств $\operatorname{rad} \Omega=\left\{X \in C^{1}(T M): \mathrm{I}_{X} \Omega=0\right\}$, где $\mathrm{I}_{X} \Omega$ обозначает $(p-1)$-форму на $M$, полученную заменой первого аргумента в $p$-форме $\Omega$ векторным полем $X$.

Теорема 5.2. На вещественном однородном римановом пространстве $M=$ $G / H$ размерности $2 n$ существует $G$-инвариантная почти паракомплексная структура тогда и только тогда, когда на $M$ существует $G$-инвариантная $n$ форма с радикалом ранга $n$.

ДокАзАтельство. Достаточно показать, что на $M$ существует $G$-инвариантное распределение $n$-мерных касательных подпространств тогда и только тогда, когда на $M$ существует $G$-инвариантная $n$-форма с радикалом ранга $n$.

Пусть $\Omega-G$-инвариантная $n$-форма на $M$ и $\operatorname{rank}(\operatorname{rad} \Omega)=n$. Пусть $D=\operatorname{rad} \Omega$. Покажем, что $D$ есть $G$-инвариантное распределение касательных подпространств на $M$.

Пусть $o-$ начальная точка однородного пространства $M$ и $v \in D(o)$. Поскольку $n$-форма $\Omega G$-инвариантна, для любого $g \in G$ имеем

$$
\mathrm{I}_{d g(v)} \Omega=\mathrm{I}_{v}(\Omega \circ d g)=\mathrm{I}_{v} \Omega=0,
$$


т. е. $d g(v) \in D(x), x=g(o)$. Поскольку отображение $d g$ - изоморфизм касательных пространств $T_{o} M$ и $T_{x} M, x=g(o)$, получаем $d g(D(o))=D(x), x=g(o)$ для любых $g \in G$.

Обратно, если $D-G$-инвариантное распределение $n$-мерных касательных подпространств на $M$, то в начальной точке $о$ существует набор линейно независимых 1-форм $\alpha_{1}, \ldots, \alpha_{n}$ таких, что

$$
D(o)=\left\{X \in T_{o} M: \alpha_{1}(X)=0, \ldots, \alpha_{n}(X)=0\right\} .
$$

Пусть $\Omega_{o}=\alpha_{1} \wedge \cdots \wedge \alpha_{n}$ и для любой точки $x=g(o), g \in G$,

$$
\Omega_{x}=\Omega_{0} \circ d g^{-1} .
$$

Пусть $e_{1}, \ldots, e_{2 n}-$ дуальный базис в $T_{o} M$, т. е. $\alpha_{k}\left(e_{k}\right)=1, \alpha_{k}\left(e_{l}\right)=0$ при $k \neq l$. Имеем $e_{n+1}, \ldots, e_{2 n} \in D(o)$. Из определения внешнего произведения 1 -форм и определения 5.1 следует, что $\Omega_{o}\left(e_{1}, \ldots, e_{n}\right)=1$, а $e_{n+1}, \ldots, e_{2 n}$ порождают $\operatorname{rad} \Omega_{o}=D(o)$. Поскольку $n$-форма $\Omega$ и распределение $D G$-инвариантны, $D(x)=\operatorname{rad} \Omega_{x}$ в любой точке $x \in M$.

Пусть $H$ - компактная нетривиальная подгруппа Ли в группе Ли $G, \mathfrak{g}$ - алгебра Ли группы $G, \Lambda^{p}(\mathfrak{g})$ - пространство всех левоинвариантных кососимметричных $p$-форм на алгебре Ли $\mathfrak{g}$ и $\operatorname{Ad}_{g}, g \in G$, - присоединенное представление элемента $g$ в алгебре Ли $\mathfrak{g}$ (см. [6]). Выберем на подгруппе $H$ левоинвариантную меру $\mu$ и определим линейный оператор усреднения $S_{H}: \Lambda^{p}(\mathfrak{g}) \rightarrow \Lambda^{p}(\mathfrak{g})$ по подгруппе $H$ такой, что для каждой $p$-формы $\Omega \in \Lambda^{p}(\mathfrak{g})$

$$
S_{H} \Omega=\frac{\int_{H}\left(\operatorname{Ad}_{x}^{*} \Omega\right) \mu_{x}}{\int_{H} \mu} .
$$

Предложение 5.3. Пусть $H$ - нетривиальная компактная связная подгруппа Ли в группе Ли $G$ и $\Omega \in \Lambda^{p}(\mathfrak{g})$. Тогда справедливы следующие утверждения:

(1) $S_{H} \Omega-H$-биинвариантная p-форма;

(2) если $\Omega-H$-биинвариантная $p$-форма, то $S_{H} \Omega=\Omega$;

(3) если $S_{H} \Omega \neq 0$, то $\operatorname{rank}\left(\operatorname{rad}\left(S_{H} \Omega\right)\right)=\operatorname{rank}(\operatorname{rad} \Omega)$.

ДокАЗАТЕЛЬСтво. Так как любая связная компактная группа Ли унимодулярна (см. [6]), на подгруппе $H$ существует биинвариантная мера $\mu$, т. е. $\operatorname{Ad}_{h}^{*} \mu=\mu$ для любых $h \in H$. Пусть $x \in H, h \in H, y=h x$. Имеем

$$
\operatorname{Ad}_{h}^{*}\left(S_{H} \Omega\right)=\frac{\int_{H}\left(\operatorname{Ad}_{h}^{*}\left(\operatorname{Ad}_{x}^{*} \Omega\right)\right) \mu_{x}}{\int_{H} \mu}=\frac{\int_{H}\left(\operatorname{Ad}_{h x}^{*} \Omega\right) \operatorname{Ad}_{h}^{*} \mu_{h x}}{\int_{H} \mu}=\frac{\int_{H}\left(\operatorname{Ad}_{y}^{*} \Omega\right) \mu_{y}}{\int_{H} \mu}=S_{H} \Omega .
$$

Это доказывает свойство (1).

Из интегральной теоремы о среднем следует, что существует элемент $h_{0} \in$ $H$ такой, что

$$
\int_{H}\left(\operatorname{Ad}_{x}^{*} \Omega\right) \mu_{x}=\operatorname{Ad}_{h_{0}}^{*} \Omega \int_{H} \mu .
$$

Отсюда $S_{H} \Omega=\operatorname{Ad}_{h_{0}}^{*} \Omega$. Если $p$-форма $\Omega H$-биинвариантна, то $\operatorname{Ad}_{h_{0}}^{*} \Omega=\Omega$. Если $S_{H} \Omega \neq 0$, то, поскольку $\operatorname{Ad}_{h_{0}}-$ автоморфизм алгебры Ли $\mathfrak{g}$, имеем $\operatorname{rad}\left(\operatorname{Ad}_{h_{0}}^{*} \Omega\right)=\operatorname{Ad}_{h_{0}}^{-1}(\operatorname{rad} \Omega)$. Это доказывает свойства $(2)$ и (3). 
Касательное пространство $T_{x}\left(S^{6}\right)$ в точке $x \in S^{6}$ изоморфно множеству мнимых октонионов $y \in \mathbb{R}^{7}: g_{0}(y, x)=0$. Заметим, что $x \cdot \bar{x}=g_{0}(x, x)=1$ для любых $x \in S^{6}$ и $\bar{y}=-y$ для любых $y \in \mathbb{R}^{7}$. Определим на $S^{6}$ непрерывное поле линейных операторов $J_{0}(x) y, x \in S^{6}, y \in T_{x}\left(S^{6}\right)$, таких, что $J_{0}(x) y=x \cdot \bar{y}$. Для любых $y \in T_{x}\left(S^{6}\right)$ имеем

$$
\begin{aligned}
g_{0}\left(J_{0}(x) y, x\right)=g_{0}(x \cdot \bar{y}, x)=-g_{0}(x \cdot y, x) & =-\operatorname{Re}(x \cdot y \cdot \bar{x}) \\
& =-\operatorname{Re}(x \cdot \bar{y} \cdot \bar{x})=-g_{0}\left(J_{0}(x) y, x\right),
\end{aligned}
$$

Отсюда $g_{0}\left(J_{0}(x) y, x\right)=0$, т. е. $J_{0}(x) y \in T_{x}\left(S^{6}\right)$. Поскольку касательное пространство $T_{x}\left(S^{6}\right)$ изоморфно подпространству мнимых октонионов, имеем $\overline{(x \cdot \bar{y})}=-x \cdot \bar{y}$. Далее,

$$
\begin{gathered}
J_{0}^{2}(x) y=x \cdot \overline{(x \cdot \bar{y})}=-x \cdot x \cdot \bar{y}=-(\bar{x} \cdot x) \cdot y=-y, \\
g_{0}\left(J_{0}(x) y, J_{0}(x) y\right)=\operatorname{Re}(x \cdot \bar{y} \cdot \overline{(x \cdot \bar{y})})=(x \cdot \bar{x})(\bar{y} \cdot y)=g_{0}(y, y) .
\end{gathered}
$$

Тем самым $J_{0}$ есть $G_{2}$-инвариантная почти комплексная структура на $S^{6}$, ортогональная относительно евклидовой метрики $g_{0}$. Фундаментальная 2-форма $\Theta$ почти эрмитовой структуры $\left(J_{0}, g_{0}\right)$ есть $G_{2}$-инвариантная невырожденная кососимметричная 2-форма на $S^{6}$, а $d \Theta-G_{2}$-инвариантная 3-форма на $S^{6}$.

Алгебра Ли $\mathfrak{g}_{2}$ группы $G_{2}$ допускает $H$-биинвариантное скалярное произведение, где $H=\mathrm{SU}(3)$ (см. [6]), и $\mathfrak{g}_{2}$ раскладывается в прямую сумму $\mathfrak{m} \oplus \mathfrak{h}$, где $\mathfrak{h}$ - алгебра Ли подгруппы $\mathrm{SU}(3), \mathfrak{m}$ - ортогональное дополнение к подалгебре $\mathfrak{h}$. Подпространство $\mathfrak{m} \mathrm{Ad}_{H}$-инвариантно. Отсюда следует, что множество всех $G_{2}$-инвариантных 3 -форм на $S^{6}$ находится во взаимно однозначном соответствии с множеством $G_{2}$-левоинвариантных $H$-правоинвариантных 3 -форм на подпространстве $\mathfrak{m}$. В частности, 3-форма $d \Theta$ порождает $H$-биинвариантную 3 форму $\eta: d \Theta=\left.\eta \circ d \pi\right|_{\mathfrak{m}}$ на подпространстве $\mathfrak{m}$, где $\pi-$ проекция $G_{2} \rightarrow S^{6}$. Из предложения 5.3 следует, что $S_{H} \eta=\eta$.

Предложение 5.4. На подпространстве $\mathfrak{m}$ существует $H$-биинвариантная 3-форма $\Omega, H=\mathrm{SU}(3)$, такая, что $S_{H} \Omega \neq 0$ и $\operatorname{rank}(\operatorname{rad} \Omega)=3$.

ДоКАЗАТЕЛЬСТвО. Пусть $\theta_{1}, \ldots, \theta_{6}-$ левоинвариантный базис пространства $\mathfrak{m}^{*}$. Тогда 3 -формы $\alpha_{i j k}=\theta_{i} \wedge \theta_{j} \wedge \theta_{k}, i<j<k \leq 6$, образуют левоинвариантный базис пространства $\Lambda^{3}(\mathfrak{m})$. Поскольку $\eta \in \Lambda^{3}(\mathfrak{m})$, существуют коэффициенты $a_{i j k}, i<j<k \leq 6$, такие, что

$$
\eta=\sum_{i<j<k} a_{i j k} \alpha_{i j k} .
$$

Так как подгруппа $H=\mathrm{SU}(3)$ есть компактная связная группа Ли, из предложения 5.3 следует, что

$$
\sum_{i<j<k} a_{i j k} S_{H} \alpha_{i j k}=S_{H} \eta=\eta .
$$

Это влечет существование хотя бы одного набора индексов $i, j, k$, для которого $S_{H} \alpha_{i j k} \neq 0$. Без потери общности можно считать, что $i=1, j=2, k=3$. Легко видеть, что $\operatorname{rank}\left(\operatorname{rad} \alpha_{123}\right)=3$. Из предложения 5.3 получаем, что 3-форма $\Omega=S_{H} \alpha_{123}$ есть $H$-биинвариантная 3 -форма на $\mathfrak{m}$ и $\operatorname{rank}(\operatorname{rad} \Omega)=3$.

Поскольку каждая $H$-биинвариантная 3 -форма на подпространстве $\mathfrak{m}$ индуцирует $G_{2}$-инвариантную 3 -форму на $S^{6}$, из теоремы 5.2 и предложения 5.4 
вытекает, что на сфере $S^{6}$ существует $G_{2}$-инвариантная почти паракомплексная структура, порожденная почти комплексной структурой на $S^{6}$. Обозначим эту почти паракомплексную структуру через $\Phi_{0}$. В $\S 2$ введено понятие интегрируемой почти паракомплексной структуры. Докажем, что почти паракомплексная структура $\Phi_{0}$ неинтегрируема.

Теорема 5.5. Пусть $M=G / H$ - однородное риманово пространство размерности 6, $G$ - простая группа Ли, транзитивно действующая на $M$, и $H$ - компактная связная подгруппа изотропии начальной точки $о$. Любая $G$ инвариантная 3-форма на $M$ с радикалом ранга 3 порождает на $M$ неинтегрируемую $G$-инвариантную почти паракомплексную структуру.

ДокАзАтельство. Пусть $\Omega-G$-инвариантная 3 -форма на $M$ такая, что $\operatorname{rank}(\operatorname{rad} \Omega)=3$. По теореме 5.23 -форма $\Omega$ определяет на $M G$-инвариантную почти паракомплексную структуру $\Phi$. Предположим, что почти паракомплексная структура $\Phi$ интегрируема. Тогда распределения собственных подпространств $D_{+}$и $D_{-}$на $M$ инволютивны.

Пусть $\mathfrak{g}$ - алгебра Ли группы $G, \mathfrak{h}$ - алгебра Ли подгруппы изотропии $H$ и $\mathfrak{m}$ - ортогональное дополнение к $\mathfrak{h}$ относительно $H$-биинвариантного скалярного произведения в $\mathfrak{g}$. Пусть $\mathfrak{M}$ есть $\operatorname{Ad}_{H}$-инвариантное подпространство и $[\mathfrak{m}, \mathfrak{h}] \subseteq \mathfrak{m}$.

Пусть $\pi-$ проекция $G \rightarrow M$. Поскольку $T_{o} M=D_{+}(o) \oplus D_{-}(o)$ и $d \pi-$ изоморфизм $\mathfrak{m} \rightarrow T_{o} M$, в $\mathfrak{m}$ существуют подалгебры $\mathfrak{m}_{+}=d \pi^{-1} D_{+}(o)$ и $\mathfrak{m}_{-}=$ $d \pi^{-1} D_{-}(o)$ такие, что $\mathfrak{m}=\mathfrak{m}_{+} \oplus \mathfrak{m}_{-}$. По теореме 2.5 в окрестности точки $o$ пространство $M$ диффеоморфно прямому произведению двух подпространств $M_{+}: T M_{+}=\left.D_{+}\right|_{M_{+}}$и $M_{-}: T M_{-}=\left.D_{-}\right|_{M_{-}}$. Отсюда следует, что скобка Ли подалгебр $\mathfrak{m}_{+}$и $\mathfrak{m}_{-}$равна 0 . Получаем, что $\mathfrak{m}$ есть подалгебра в $\mathfrak{g}$. Из разложения $\mathfrak{g}=\mathfrak{m} \oplus \mathfrak{h}$ следует

$$
[\mathfrak{g}, \mathfrak{m}]=[\mathfrak{m}, \mathfrak{m}] \oplus[\mathfrak{h}, \mathfrak{m}] \subseteq \mathfrak{m},
$$

т. е. $\mathfrak{m}$ - идеал в $\mathfrak{g}$. Поскольку алгебра Ли $\mathfrak{g}$ простая, в $\mathfrak{g}$ не может существовать нетривиальных идеалов. Таким образом, почти паракомплексная структура $\Phi$ не может быть интегрируемой.

Поскольку группа $G_{2}$ проста, из теоремы 5.5 следует, что полученная выше почти паракомплексная структура $\Phi_{0}$ на $S^{6}$ неинтегрируема. Более того, из доказательства теоремы 5.5 следует, что любая $G_{2}$-инвариантная почти паракомплексная структура на $S^{6}$ неинтегрируема.

Почти паракомплексная структура $\Phi_{0}$ есть глобальное сечение расслоения $\mathscr{O}\left(S^{6}\right)$ ортогональных паракомплексных структур над $S^{6}$. Применяя следствие 3.2 , получаем

Следствие 5.6. Расслоение $\mathscr{O}\left(S^{6}\right)$ ортогональных паракомплексных структур над шестимерной сферой изоморфно расслоению $\operatorname{gr}^{3}\left(S^{6}\right) \times \mathbb{Z}_{2}$, где $\operatorname{gr}^{3}\left(S^{6}\right)$ является расслоением 3-грассманианов над $S^{6}$ и допускает глобальное сечение.

Структурой почти произведения типа $(p, q)$ на вещественном многообразии $M$ размерности $n$ называется непрерывное поле $\psi$ автоморфизмов касательных пространств такое, что $\psi^{2}=\mathrm{id}, \operatorname{rank}\left(D_{+}\right)=p, \operatorname{rank}\left(D_{-}\right)=q, p+q=n$, где $D_{+}-$распределение собственных подпространств для собственного значения 1 , $D_{-}$- распределение собственных подпространств для собственного значения -1. Почти паракомплексная структура на $M$ является структурой почти произведения типа $(p, p)$. Используя фундаментальную 2-форму полученной выше 
почти эрмитовой структуры на $S^{6}$, таким же способом, как при построении $G_{2}$ инвариантной почти паракомплексной структуры на $S^{6}$, на $S^{6}$ можно получить $G_{2}$-инвариантную кососимметричную 2 -форму $\Omega: \operatorname{rank}(\operatorname{rad} \Omega)=4$. Полагая $D_{+}=\operatorname{rad} \Omega, D_{-}=(\operatorname{rad} \Omega)^{\perp}$, получаем на $S^{6} g_{2}$-инвариантную структуру почти произведения типа $(4,2)$. Полагая $D_{+}=(\operatorname{rad} \Omega)^{\perp}, D_{-}=\operatorname{rad} \Omega$, получаем на $S^{6} G_{2}$-инвариантную структуру почти произведения типа $(2,4)$. Обе эти структуры почти произведения неинтегрируемы, т. е. распределения $D_{+}$и $D_{-}$ неинволютивны. Поскольку на $S^{6}$ любое векторное поле обращается в 0 хотя бы в одной точке, на $S^{6}$ не существует структур почти произведения типа $(1,5)$ или $(5,1)$.

\section{ЛИТЕРАТУРА}

1. Cruceanu V., Fortuny P., Gadea P. M. A survey on paracomplex geometry // Rocky Mountain J. Math. 1996. V. 26, N 1. P. 83-115.

2. Алексеевский Д. В., Медори К., Томассини А. Однородные пара-кэлеровы многообразия Эйнштейна // Успехи мат. наук. 2009. Т. 64, № 1. С. 3-50.

3. Смоленцев H. К. О почти (пара)комплексных структурах Кэли на сферах $S^{2,4}$ и $S^{3,3} / /$ Вестн. Том. гос. ун-та. Математика и механика. 2018. Т. 53. С. 22-38.

4. Корнев Е. С. Субкомплексные и субкэлеровы структуры // Сиб. мат. журн. 2016. Т. 57, № 5. С. 1062-1077.

5. Кобаяси Ш., Номидзу К. Основы дифференциальной геометрии (В 2 т.). М.: Наука, 1981.

6. Бессе А. Многообразия Эйнштейна (В 2 т.). М.: Мир, 1990.

7. Милнор Дж., Сташеф Дж. Характеристические классы. М.: Мир, 1979.

8. Сергеев А. Г. Гармонические отображения. М.: МИАН, 2008. (Лекционные курсы НОЦ; вып. 10).

Поступила в редакцию 9 декабря 2019 г.

После доработки 9 января 2020 г.

Принята к публикации 19 февраля 2020 г.

Корнев Евгений Сергеевич

Кемеровский государственный университет,

ул. Красная, 6, Кемерово 650043

q148@mail.ru 